\title{
Casimir energy in multiply connected static hyperbolic universes
}

\author{
Daniel Müller* \\ Instituto de Física, Universidade de Brasília, Campus Universitário Darcy Ribeiro, Caixa Postal 04455, CEP 70919-970, \\ Brasília, DF, Brazil \\ Helio V. Fagundes ${ }^{\dagger}$ \\ Instituto de Física Teórica, Universidade Estadual Paulista Rua Pamplona, 145, CEP 01405-900, São Paulo, SP, Brazil \\ Reuven Opher ${ }^{\ddagger}$ \\ Instituto de Astronomia, Geofísica e Ciências Atmosféricas, Universidade de São Paulo Rua do Matão, 1226, Cidade Universitária, \\ CEP 05508-900, São Paulo, SP, Brazil
}

(Received 17 July 2002; published 18 October 2002)

\begin{abstract}
We generalize a previously obtained result for the case of a few other static hyperbolic universes with manifolds of nontrivial topology as spatial sections.

DOI: 10.1103/PhysRevD.66.083507

PACS number(s): 98.80.Cq, 98.80.Hw
\end{abstract}

\section{INTRODUCTION}

As is well known, Einstein equations (EQ) restrict the local geometry of spatially homogeneous and isotropic spacetimes to those of $R^{3}, S^{3}$, or $H^{3}$. Recent observational data indicate that the curvature of the universe is small, without ruling out the case of negative curvature. On the other hand, the EQ are insensitive to a global nontrivial topology of space, which can be a compact hyperbolic 3-space $\mathcal{M}$, which is isometric to a quotient space $H^{3} / \Gamma$. Here $\Gamma$ is a nontrivial discrete group of isometries (known as the holonomy group), which acts freely and properly discontinuously on the covering space $H^{3}$. Also important is the fact that $\Gamma$ is isomorphic to the fundamental group $\pi_{1}(\mathcal{M})$, which is a group of homotopy classes of maps of the circle $S^{1}$ into $\mathcal{M}$ [1]. Since $\pi_{1}(\mathcal{M})$ is nontrivial, $\mathcal{M}$ is multiply connected.

The 3-space $\mathcal{M}$ may be represented by a fundamental polyhedron $F P$ in $H^{3}$, with an even number of faces, whose copies $\gamma(F P), \gamma \in \Gamma$, fill up the entire $H^{3}$. The faces of $F P$ are pairwise identified by the basic elements, or generators, of $\Gamma$. The resulting manifold is a bundle with discrete fibers $\Gamma p$ over base points $p$ in the fundamental polyhedron. These fibers are the points of the quotient space.

Among the first applications of the topology considerations, there was an attempt to explain multiple quasar images [2]. For recent reviews of topology in connection with cosmology, see [3,4], and the article [5] for compactifications of the 3 -sphere.

The first astrophysical limits on the topology of the universe were obtained for a 3-torus $T^{3}$. Accordance with the homogeneity of the cosmic microwave background radiation (CMBR) puts a lower limit on the size of the fundamental cell, about $3000 \mathrm{Mpc}$, which is a cube in the cases of [6] and [7]. Later on, it was shown that this result is very sensitive to

\footnotetext{
*Electronic address: muller@ fis.unb.br

†Electronic address: helio@ift.unesp.br

${ }^{\ddagger}$ Electronic address: opher@astro.iag.usp.br
}

the type of compactification of the spatial sections. For a universe with spatial sections $T^{2} \times R$, the fundamental cell's size is about $1 / 10$ of the horizon, and is compatible with the homogeneity of the CMBR [8].

In compact universes, the pair separation histogram would present spikes for characteristic distances. At first it was thought that this technique, known as the crystallographic method, was able to totally determine the topology of the universe [9]. It turned out that the crystallographic method only applied when the holonomy group contained at least a Clifford translation, i.e. a translation which moves all the points by the same distance [10] and [11]. Generalizations of the crystallographic method were proposed, for example in [12].

Also in compact universes the light front of the CMBR interacts with itself producing circles in its sky pattern [13].

A recent result has called our attention to the possibility that methods based on multiple images will prove not to be efficient [14]. The reasoning is that, according to observations, the curvature is very small, so the fundamental regions are so big that there has not been time enough for the formation of ghost images. The result is that for low curvature universes such as ours, only compact universes with the smallest volumes could be detected by pattern repetitions.

A very attractive argument in favor of compact hyperbolic manifolds is related to pre-inflationary homogenization through chaotic mixing [15]. The effect is the same that arises in compact hyperbolic surfaces. The geodesic motion on a surface of genus $g>1$ shows the absence of KAM torus [16]. Not only is it ergodic but it also satisfies the Anosov property, which indicates the presence of strong chaos [16]. The chaotic properties of the $g=2$ torus were previously studied, for example in [17].

We extend our previously obtained result [18] (see also [19]) for a few more compact hyperbolic universes. In [18] we numerically calculated the Casimir effect of a scalar field for a static universe whose spatial section is the Weeks manifold, the smallest volume (with curvature normalized to $K$ $=-1$ ) compact hyperbolic manifold known. The outcome 
of our calculation is in fact a Casimir energy density $\rho_{C}$.

In compactifications of flat space, the strictly speaking Casimir energy can be analytically obtained through the very elegant zeta functions techniques; for a recent review see [20]. Unfortunately this formalism does not yield an analytical result in the case of hyperbolic compact manifolds, since the spectrum of the Laplace-Beltrami operator can only be determined numerically.

We use the point splitting technique in the covering space, which is a static hyperbolic universe. The obtained propagator is exact and possesses information about the global properties of the manifold, in the sense that the infrared modes are taken into account.

When the spacetime is multiply connected, the propagator is obtained as the usual sum over paths: all geodesics connecting the two points are taken into account.

We find a static hyperbolic solution for the EQ in Sec. II. In Sec. III we write the expression for the vacuum expectation value of the energy-momentum tensor in compact hyperbolic universes. We obtain in Sec. IV the numerical values of the Casimir energy density in a few multiply connected static spacetimes. Our conclusions are presented in Sec. V. (We use natural units, $G=c=\hbar=1$, except in Sec. II.)

\section{QUANTUM FIELD THEORY IN THE STATIC UNIVERSE $R \times H^{3}$}

The hyperbolic space sections $H^{3}$, can be realized as a surface

$$
\left(x-x^{\prime}\right)^{2}+\left(y-y^{\prime}\right)^{2}+\left(z-z^{\prime}\right)^{2}-\left(w-w^{\prime}\right)^{2}=-a^{2},
$$

imbedded in a Minkowski 4-space

$$
d l^{2}=d x^{2}+d y^{2}+d z^{2}-d w^{2} .
$$

As this space is homogeneous, we explicitly write the origin of coordinates $\left(x^{\prime}, y^{\prime}, z^{\prime}, w^{\prime}\right)$. It can easily be seen that its isometry group is the proper, orthocronous Lorentz group $S O^{\uparrow}(1,3)$, which is isomorphic to $\operatorname{PSL}(2, C)=\operatorname{SL}(2, C)$ / $\{ \pm I\}[21]$. With the constraint of Eq. (1) on the line element we obtain

$$
\begin{aligned}
d l^{2}= & d x^{2}+d y^{2}+d z^{2} \\
& -\frac{\left[\left(x-x^{\prime}\right) d x+\left(y-y^{\prime}\right) d y+\left(z-z^{\prime}\right) d z\right]^{2}}{\left(x-x^{\prime}\right)^{2}+\left(y-y^{\prime}\right)^{2}+\left(z-z^{\prime}\right)^{2}+a^{2}}, \\
d s^{2}= & -d t^{2}+d l^{2}=g\left(x, x^{\prime}\right)_{\mu \nu} d x^{\mu} d x^{\nu},
\end{aligned}
$$

where we interchangeably write $\left(x^{0}, x^{1}, x^{2}, x^{2}\right) \leftrightarrow(t, x, y, z)$. Both connections, $\nabla_{x}$ and $\nabla_{x^{\prime}}$, compatible with the metric of Eq. (2), can be defined through

$$
\begin{gathered}
\nabla_{\mu} g\left(x, x^{\prime}\right)_{\alpha \beta} \equiv 0, \\
\nabla_{\mu^{\prime}} g\left(x, x^{\prime}\right)_{\alpha \beta} \equiv 0 .
\end{gathered}
$$

The expression in Eq. (2) is the popular Robertson-Walker line element, which written in the Lobatchevsky form reads

$$
d s^{2}=-d t^{2}+a^{2}\left[d \chi^{2}+\sinh ^{2} \chi\left(d \theta^{2}+\sin ^{2} \theta d \phi^{2}\right)\right],
$$

with

$$
\sinh ^{2} \chi=\frac{\left(x-x^{\prime}\right)^{2}+\left(y-y^{\prime}\right)^{2}+\left(z-z^{\prime}\right)^{2}}{a^{2}}
$$

As is well known, the EQ for the homogeneous and isotropic space sections in Eq. (5), with $a=a(t)$, reduce to the Friedmann-Lemaitre equations

$$
\begin{array}{r}
\left(\frac{\dot{a}}{a}\right)^{2}-\frac{1}{a^{2}}=\frac{8 \pi G}{3} \rho+\frac{\Lambda}{3}, \\
2\left(\frac{\ddot{a}}{a}\right)+\left(\frac{\dot{a}}{a}\right)^{2}-\frac{1}{a^{2}}=-8 \pi G p+\Lambda,
\end{array}
$$

where the right-hand side comes from the classical energymomentum source for the geometry, $T^{\mu \nu}=(\rho+p) u^{\mu} u^{\nu}$ $+p g^{\mu \nu}$, plus the cosmological constant term $\Lambda g^{\mu \nu}$.

We assume that the universe was radiation dominated near the Planck era, hence $p=\rho / 3$, and we obtain the following static solution:

$$
\begin{aligned}
a & =\sqrt{\frac{3}{2|\Lambda|},} \\
\rho & =\frac{\Lambda}{8 \pi G}, \\
d s^{2} & =-d t^{2}+a^{2}\left[d \chi^{2}+\sinh ^{2} \chi\left(d \theta^{2}+\sin ^{2} \theta d \phi^{2}\right)\right],
\end{aligned}
$$

where the cosmological constant is negative.

We now wish to evaluate the vacuum expectation value of the energy density for the case of a universe consisting of a classical radiation fluid, a cosmological constant, and a noninteracting quantum scalar field $\phi$. The solution of EQ is given in Eq. (6), where the quantum back reaction is disregarded. We use the point splitting method in the universal covering space $R \times H^{3}$, for which the propagator is exact. The point splitting method was constructed to obtain the renormalized (finite) expectation values of the quantum mechanical operators. It is based on the Schwinger formalism [22], and was developed in the context of curved space by DeWitt [23]. Further details are contained in the articles by Christensen [24,25]. For a review, see [26].

Metric variations in the scalar action

$$
S=-\frac{1}{2} \int \sqrt{-g}\left(\phi_{, \rho} \phi^{, \rho}+\xi R \phi^{2}+m^{2} \phi^{2}\right) d^{4} x,
$$

with conformal coupling $\xi=1 / 6$, give the classical energymomentum tensor 


$$
\begin{aligned}
T_{\mu \nu}= & \frac{2}{3} \phi_{, \mu} \phi_{, \nu}-\frac{1}{6} \phi_{, \rho} \phi^{, \rho} g_{\mu \nu}-\frac{1}{3} \phi \phi_{; \mu \nu}+\frac{1}{3} g_{\mu \nu} \phi \square \phi \\
& +\frac{1}{6} G_{\mu \nu} \phi^{2}-\frac{1}{2} m^{2} g_{\mu \nu} \phi^{2},
\end{aligned}
$$

where $G_{\mu \nu}$ is the Einstein tensor. As expected for massless fields, it can be verified that the trace of the above tensor is identically zero if $m=0$. Variations with respect to $\phi$ result in the curved space generalization of the Klein-Gordon equation,

$$
\square \phi-\frac{R}{6} \phi-m^{2} \phi=0 .
$$

The renormalized energy-momentum tensor involves field products at the same spacetime point. Thus the idea is to calculate the averaged products at separate points, $x$ and $x^{\prime}$, taking the limit $x^{\prime} \rightarrow x$ in the end:

$$
\left\langle 0\left|T_{\mu \nu}(x)\right| 0\right\rangle=\lim _{x^{\prime} \rightarrow x} T\left(x, x^{\prime}\right)_{\mu \nu},
$$

with

$$
\begin{aligned}
T\left(x, x^{\prime}\right)_{\mu \nu}= & {\left[\frac{1}{6}\left(\nabla_{\mu} \nabla_{\nu^{\prime}}+\nabla_{\mu} \nabla_{\nu}\right)-\frac{1}{12} g(x)_{\mu \nu} \nabla_{\rho} \nabla^{\rho^{\prime}}-\frac{1}{12}\left(\nabla_{\mu} \nabla_{\nu}+\nabla_{\mu^{\prime}} \nabla_{\nu^{\prime}}\right)\right.} \\
& \left.+\frac{1}{48} g(x)_{\mu \nu}\left(\square+\square^{\prime}\right)+\frac{1}{12}\left(R(x)_{\mu \nu}-\frac{1}{4} R(x) g(x)_{\mu \nu}\right)-\frac{1}{8} m^{2} g(x)_{\mu \nu}\right] G^{(1)}\left(x, x^{\prime}\right),
\end{aligned}
$$

where the covariant derivatives are defined in Eqs. (3) and (4), and $G^{(1)}$ is the Hadamard function, which is the expectation value of the anti-commutator of $\phi(x)$ and $\phi\left(x^{\prime}\right)$ (see below). We stress that the quantity $T\left(x, x^{\prime}\right)_{\mu \nu}$ only makes sense after the limit in Eq. (9) is taken. The geometric quantities such as the metric and the curvature are regarded as classical entities. $g(x)_{\mu \nu}=g\left(x, x^{\prime}=0\right)_{\mu \nu}$ is obtained from the line element in Eq. (2).

The causal Green function, of Feynman propagator, is obtained as

$$
G\left(x, x^{\prime}\right)=i\left\langle 0\left|T \phi(x) \phi\left(x^{\prime}\right)\right| 0\right\rangle,
$$

where $T$ is the time-ordering operator. Taking its real and imaginary parts,

$$
G\left(x, x^{\prime}\right)=G_{s}\left(x, x^{\prime}\right)+\frac{i}{2} G^{(1)}\left(x, x^{\prime}\right),
$$

we get, for the Hadamard function,

$$
G^{(1)}\left(x, x^{\prime}\right)=\left\langle 0\left|\left\{\phi(x), \phi\left(x^{\prime}\right)\right\}\right| 0\right\rangle=2 \operatorname{Im} G\left(x, x^{\prime}\right) .
$$

\section{THE FEYNMAN PROPAGATOR AND THE CASIMIR ENERGY DENSITY IN $R \times \mathcal{M}$}

Green functions, as any other function defined in the spatially compact spacetime $R \times \mathcal{M}$, must have the same periodicities of the manifold $\mathcal{M}$ itself. One way of imposing this periodicity is by determining the spectrum of the Laplacian, which can only be done numerically.

Another method imposes the periodicity by brute force:

$$
f_{\mathcal{M}}(x)=\sum_{\gamma \in \Gamma} f(\gamma x)
$$

The above expression is named the Poincare series, and when it converges, it defines functions $f_{\mathcal{M}}$ on the manifold $\mathcal{M}$.

We define the operator

$$
F\left(x, x^{\prime}\right)=F(x) / \sqrt{-g} \delta\left(x-x^{\prime}\right),
$$

where $F(x)=\square-R / 6-m^{2}$, and introduce an auxiliary evolution parameter $s$ and a complete orthonormal set of states $|x\rangle$, such that

$$
\begin{aligned}
G\left(x, x^{\prime}\right) & =\left\langle x|\hat{G}| x^{\prime}\right\rangle, \\
F\left(x, x^{\prime}\right) & =\left\langle x|\hat{F}| x^{\prime}\right\rangle, \\
\hat{G} & =i \int_{0}^{\infty} e^{-i s \hat{F}} d s .
\end{aligned}
$$

This last equation implies that $\hat{G}=(\hat{F}-i 0)^{-1}$, hence the causal Green function becomes

$$
G\left(x, x^{\prime}\right)=i \int_{0}^{\infty} d s\left\langle x|\exp (-i s \hat{F})| x^{\prime}\right\rangle,
$$

and the matrix element $\left\langle x|\exp (-i s \hat{F})| x^{\prime}\right\rangle=\left\langle x(s) \mid x^{\prime}(0)\right\rangle$ satisfies a Schrödinger-type equation,

$$
i \frac{\partial}{\partial s}\left\langle x(s) \mid x^{\prime}(0)\right\rangle=\left(\square-\frac{R}{6}-m^{2}\right)\left\langle x(s) \mid x^{\prime}(0)\right\rangle .
$$

Assuming that $\left\langle x(s) \mid x^{\prime}(0)\right\rangle$ depends only on the total geodesic distance $-\left(t-t^{\prime}\right)^{2}+a^{2} \chi^{2}$, with the spatial part $a^{2} \chi^{2}$ derived from Eq. (5), the above equation can be solved, and we get 


$$
\left\langle x(s) \mid x^{\prime}(0)\right\rangle=\frac{-i \chi}{\sinh \chi} \frac{\exp \left\{i m^{2} s+i\left[\left(t-t^{\prime}\right)^{2}-a^{2} \chi^{2}\right] / 4 s\right\}}{(4 \pi s)^{2}} .
$$

Substituting this solution for the integrand in Eq. (14) gives for the Feynman propagator

$$
G\left(x, x^{\prime}\right)=-\frac{m}{8 \pi} \frac{\chi}{\sinh \chi} \frac{H_{1}^{(2)}\left(m \sqrt{\left(t-t^{\prime}\right)^{2}-a^{2} \chi^{2}}\right)}{\sqrt{\left(t-t^{\prime}\right)^{2}-a^{2} \chi^{2}}},
$$

where $H_{1}^{(2)}$ is the Hankel function of the second kind and order one.

The Hadamard function can be obtained from Eqs. (16) and (11),

$$
G^{(1)}\left(x, x^{\prime}\right)=\frac{m}{2 \pi^{2}} \frac{\chi}{\sinh \chi} \frac{K_{1}\left(m \sqrt{-\left(t-t^{\prime}\right)^{2}+a^{2} \chi^{2}}\right)}{\sqrt{-\left(t-t^{\prime}\right)^{2}+a^{2} \chi^{2}}},
$$

where $K_{1}$ is the modified Bessel function of the second kind and order one. The massless limit $m=0$ can immediately be checked:

$$
G^{(1)}\left(x, x^{\prime}\right)_{m=0}=\frac{\chi}{2 \pi^{2} \sinh (\chi)}\left\{\frac{1}{-\left(t-t^{\prime}\right)^{2}+a^{2} \chi^{2}}\right\} .
$$

Remembering that, for $a \rightarrow \infty$,

$$
\sinh \chi=a^{-1} \sqrt{\left(x-x^{\prime}\right)^{2}+\left(y-y^{\prime}\right)^{2}+\left(z-z^{\prime}\right)^{2}} \rightarrow \chi,
$$

the well known Minkowski result is recovered for the massive and massless cases,

$$
\begin{aligned}
G^{(1)}\left(x, x^{\prime}\right) & =\frac{m}{2 \pi^{2}} \frac{K_{1}\left(m \sqrt{-\left(t-t^{\prime}\right)^{2}+r^{2}}\right)}{\sqrt{-\left(t-t^{\prime}\right)^{2}+r^{2}}}, \\
G^{(1)}\left(x, x^{\prime}\right)_{m=0} & =\frac{1}{2 \pi^{2}}\left\{\frac{1}{-\left(t-t^{\prime}\right)^{2}+r^{2}}\right\},
\end{aligned}
$$

where $r$ is the geodesic distance in the spatial Euclidean section.

Substituting Eq. (17) and the covariant derivatives (3) and (4) into Eq. (10), we obtain $T\left(x, x^{\prime}\right)_{\mu \nu}$.

The Klein-Gordon equation remains unchanged under isometries:

$$
£_{\xi}\left[\left(\square-\frac{R}{6}-m^{2}\right) \phi\right]=\left(\square-\frac{R}{6}-m^{2}\right) £_{\xi} \phi,
$$

where $£_{\xi}$ is the Lie derivative with respect to the Killing vector $\xi$ that generates the isometry, hence summations in the Green functions over the discrete elements of the group $\Gamma$ is well defined.

In $\mathcal{M}=H^{3} / \Gamma$, a summation over the infinite number of geodesics connecting $x$ and $x^{\prime}$ is obtained by the action of the elements $\gamma \in \Gamma$, which are the generators $g_{i}$ and their products (except the identity; see below), on $x^{\prime}$. Since $\Gamma$ is isomorphic to $\pi_{1}(\mathcal{M})$ - see Sec. I-each geodesic linking $x$ and $x^{\prime}$ in $\mathcal{M}$ is lifted to a unique geodesic linking $x$ and $\gamma x^{\prime}$ in $H^{3}$. Thus from Eq. (9) we get

$$
\begin{aligned}
\rho_{C} & =\left\langle 0\left|T(x)_{\mu \nu}\right| 0\right\rangle_{\mathcal{M}} u^{\mu} u^{\nu} \\
& =u^{\mu} u^{\nu} \lim _{x^{\prime} \rightarrow x} \sum_{\gamma \neq 1} T\left(x, \gamma x^{\prime}\right)_{\mu \nu} .
\end{aligned}
$$

The infinite summation occurs because the spacetime $R$ $\times \mathcal{M}$ is static, so there has been enough time for the quantum interaction of the scalar field with the geometry to travel any distance. Since we know the universe is expanding, the infinite summation is not physically valid. The presence of the mass term, however, naturally introduces a cutoff.

In Eq. (18) the subscript $\gamma \neq 1$ means that the direct path is not to be taken into account. We shall show, following [26], that this exclusion is indeed equivalent to a renormalization of the cosmological constant.

First remember that the effective action $W$ is given by

$$
e^{i W}=\int \mathcal{D} \phi \exp (i S)
$$

where the action for the scalar field can be transformed into a Gaussian type after integration by parts,

$$
S=-\frac{1}{2} \int d^{4} x \sqrt{-g(x)} \int d^{4} y \sqrt{-g(y)} \phi(x) F(x, y) \phi(y) .
$$

In a very informal way, the functional integration can be regarded as a usual Gaussian integral ${ }^{1}$ which, making use of Eqs. (12) and (13), results in

$$
\begin{aligned}
e^{i W} & =\left[\operatorname{det} \frac{i \hat{F}}{2 \pi}\right]^{-1 / 2} \\
W & =\frac{i}{2} \ln [\operatorname{det} \hat{F}]+\text { const. } \\
\delta W & =\frac{i}{2} \operatorname{tr} \delta(\ln \hat{F})=\frac{i}{2} \operatorname{tr}\left[\hat{F}^{-1} \delta \hat{F}\right] \\
\delta W & =\frac{i}{2} \operatorname{tr}\left[i \int_{0}^{\infty} e^{-i s \hat{F}} \delta \hat{F} d s\right] \\
\delta W & =\delta\left[-\frac{i}{2} \operatorname{tr}\left(\int_{0}^{\infty} \frac{e^{-i s \hat{F}}}{s} d s\right)\right]
\end{aligned}
$$

\footnotetext{
${ }^{1}$ This result is made more rigourous by assuming a particular complete representation basis for the operator $\hat{F}$.
} 


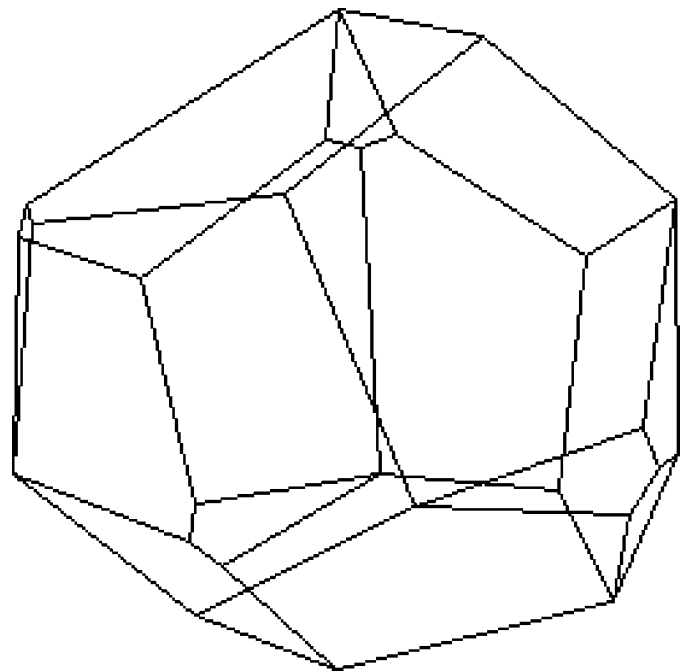

FIG. 1. Fundamental region for the Weeks manifold.

$$
W=-\frac{i}{2} \operatorname{tr}\left(\int_{0}^{\infty} \frac{e^{-i s \hat{F}}}{s} d s\right)=\int d^{4} x \mathcal{L}_{\mathrm{eff}} .
$$

The trace of the operator is over the Hilbert space, defined in Eq. (13), $\operatorname{tr} \hat{A}=\lim _{x^{\prime} \rightarrow x} \int \sqrt{-g} d^{4} x\left\langle x|\hat{A}| x^{\prime}\right\rangle$. Using the Schrödinger kernel $\left\langle x|\exp (-i s \hat{F})| x^{\prime}\right\rangle$, given in Eq. (15), we obtain the effective Lagrangian

$$
\begin{aligned}
\mathcal{L}_{\text {eff }} & =\frac{-i}{2} \sqrt{-g} \lim _{x^{\prime} \rightarrow x} \int_{0}^{\infty} \frac{\left\langle x|\exp (-i s \hat{F})| x^{\prime}\right\rangle}{s} d s \\
& =-\frac{1}{2} \sqrt{-g} \int_{0}^{\infty} \frac{e^{i m^{2} s}}{(4 \pi)^{2} s^{3}} d s=\Lambda_{\infty} \sqrt{-g},
\end{aligned}
$$

which shows that the direct path $(\gamma=1)$ corresponds to a divergent cosmological term $\Lambda_{\infty}$.

\section{CASIMIR DENSITY $\rho_{C}$ IN A FEW UNIVERSES}

According to quantum cosmology, a smaller universe has a greater probability of being spontaneously created. Also, the chaotic mixing becomes more significant for smaller volumes [15]. We describe some spatially compact universes, with increasing volumes, in Secs. IV A-IV D. As seen in Sec. I, manifolds $\mathcal{M} \cong H^{3} / \Gamma$, where $\Gamma$ is a discrete subgroup of isometries and $H^{3}$ is its universal covering, are multiply connected.

The values of $\rho_{C}$ shown for each manifold were taken at points $(\theta, \varphi)$ on the surface of a sphere inside its fundamental region. For all of them the radius of the sphere is the same, $d=a \chi=0.390035 \ldots a$, where $d$ is the geodesic distance. Our result is displayed in Figs. 2, 4, 6 and 8 for a scalar field with mass $m=0.4$, and a metric scale factor $a$ $=10$. Angles $\theta$ and $\varphi$ correspond to the co-latitude and longitude, so the lines $\theta=0$ and $\theta=\pi$ correspond to the poles of the chosen sphere. For each manifold we also write ex-

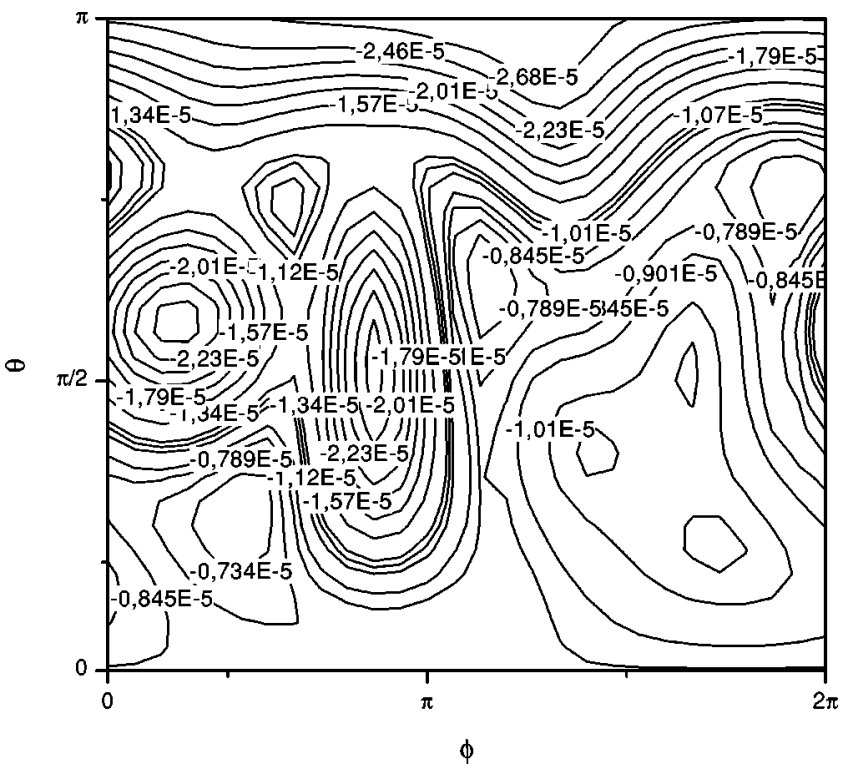

FIG. 2. $\rho_{C}$ for the Weeks universe.

plicitly the radius of the inscribed sphere $R_{\text {inradius }}$.

The description that follows applies to Secs. IV A-IV D. The $g_{i}$ matrices that generate $\Gamma$ were obtained with the computer program SNAPPEA [27]. The numerical code has been improved since our previous paper [18]. To yield a numerical result, the infinite summation (18) has to be truncated. Recall that this summation occurs in the covering space $H^{3}$. We halted the summation each time the action of the generators $g_{i}$ and their products on the origin $\left(x^{1}, x^{2}, x^{3}\right)=(0,0,0)$ reached a geodesic distance bigger than $d=a \chi$ $=5.29834 \ldots a$. Care was taken so that no point was summed more than once. In other words, the summation (18), which yelds $\rho_{C}$, was truncated when the interior of the hyperbolic sphere of geodesic radius $d=a \chi$ $=5.29834 \ldots a$, was covered with replicas of the fundamental region. We checked that additional contributions were unimportant for the evaluation of $\rho_{C}$.

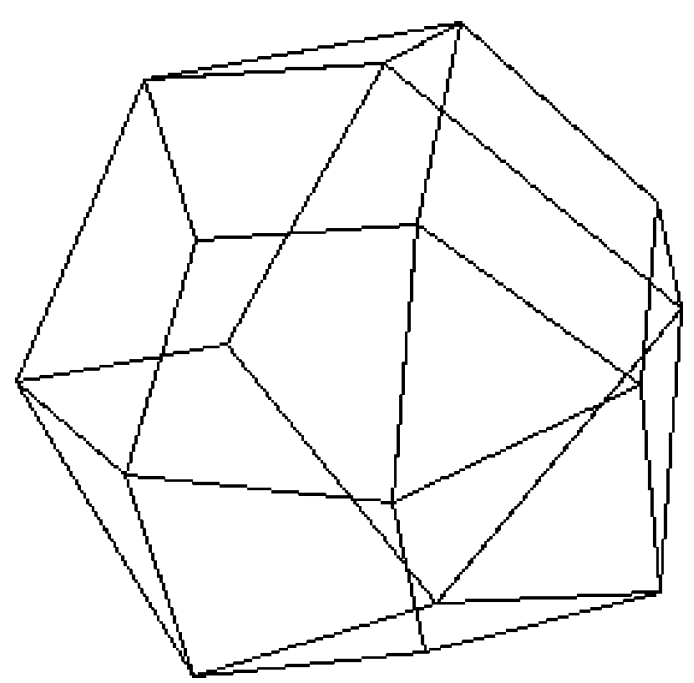

FIG. 3. Fundamental region for the Thurston manifold. 


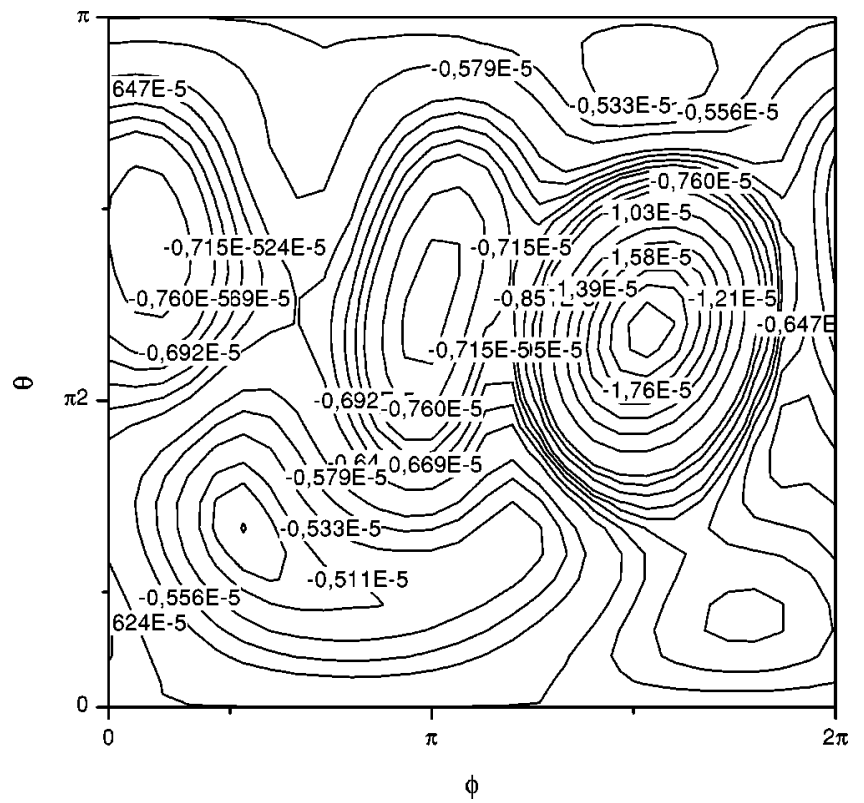

FIG. 4. $\rho_{C}$ for the Thurston universe.

\section{A. Weeks manifold}

This manifold was discovered independently by Weeks [28] and Matveev-Fomenko [29], and is the manifold with the smallest volume (in units of $a^{3}$ ) known, $V$ $=0.942707 \ldots a^{3}$. Its fundamental region is an 18 -face polyhedron, shown in Fig. 1. The radius of the inscribed sphere is $R_{\text {in radius }}=0.519162 \ldots a$.

The vacuum expectation value of the 00 -component of the energy-momentum tensor, $\rho_{C}=T_{\mu \nu} u^{\mu} u^{\nu}$, as seen by a comoving observer, is shown in Fig. 2.

\section{B. Thurston manifold}

The Thurston manifold was discovered by the field medalist William Thurston [30]. This manifold possesses a fun-

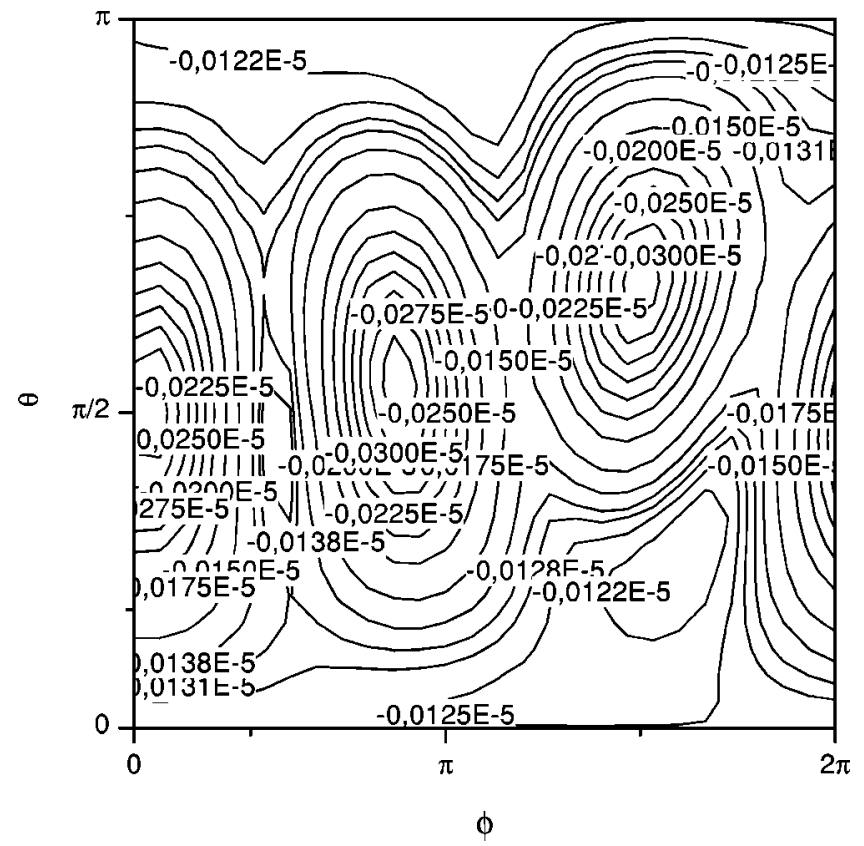

FIG. 6. $\rho_{C}$ for the Best universe.

damental region of 16 faces, its volume is $V$ $=0.981369 \ldots a^{3}$ (Fig. 3), and $R_{\text {inradius }}=0.535437 \ldots a$.

Figure 4 shows the value of $\rho_{C}=T_{\mu \nu} u^{\mu} u^{\nu}=T_{00}$, as seen by a comoving observer.

\section{Best manifold}

This manifold was discovered as a by-product of a study of finite subgroups of $S O(1,3)$ by a geometrical aproach [31]. Its fundamental region is an icosahedron with $V$ $=4.686034 \ldots a^{3}$ and $R_{\text {inradius }}=0.868298 \ldots a$, shown in Fig. 5.

The vacuum expectation value of $\rho_{C}=T_{\mu \nu} u^{\mu} u^{\nu}$, as seen by a comoving observer, is shown in Fig. 6 .

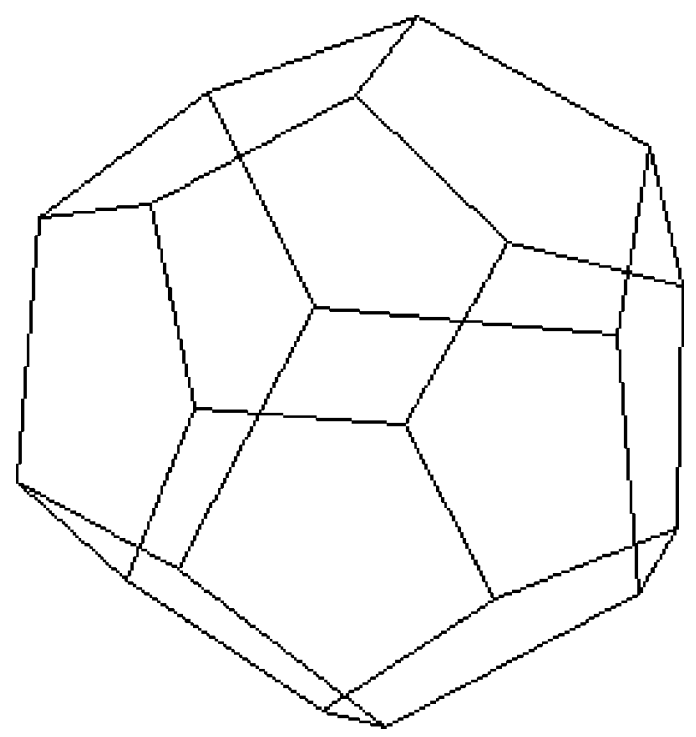

FIG. 7. Fundamental region for the Seifert-Weber manifold.

FIG. 5. Fundamental region for the Best manifold. 


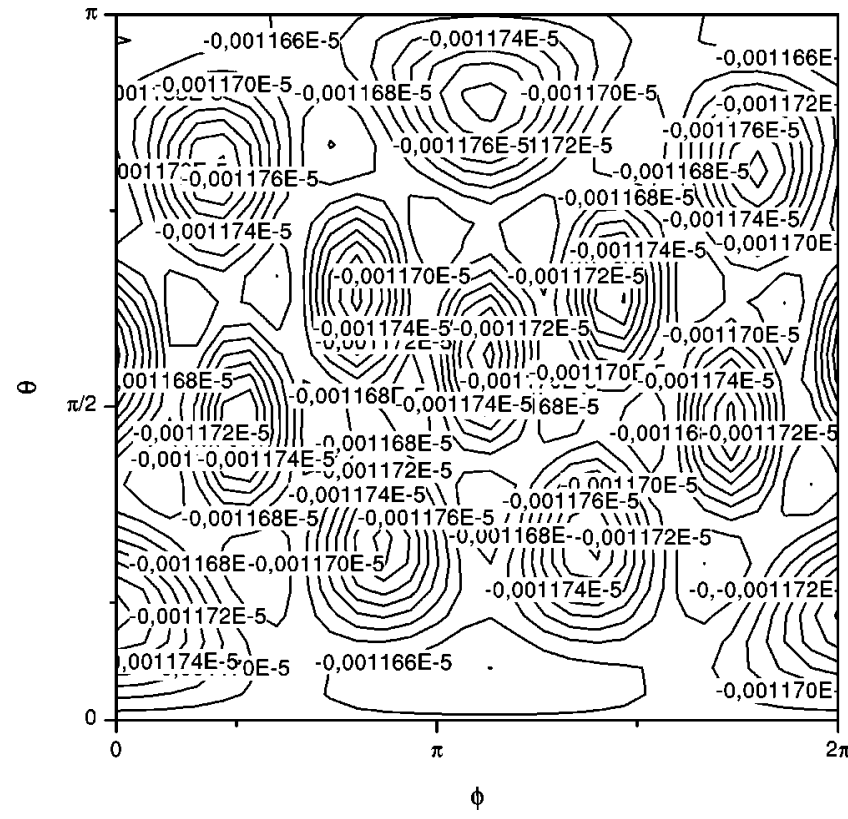

FIG. 8. $\rho_{C}$ for the Seifert-Weber universe.

\section{Seifert-Weber Manifold}

For this manifold, which was discovered by Weber and Seifert [32], $\quad V=11.199065 \ldots a^{3}, \quad R_{\text {inradius }}$ $=0.996384 \ldots a$, and the fundamental region is a dodecahedron (Fig. 7).
Figure 8 shows the value of $\rho_{C}=T_{\mu \nu} u^{\mu} u^{\nu}$, as seen by a comoving observer.

\section{CONCLUSIONS}

We explicitly evaluated the distribution of the vacuum energy density of a conformally coupled massive scalar field, for static universes with compact spatial sections of negative curvature and increasing volume: Weeks, Thurston, Best, and Seifert-Weber manifolds. As a specific example, we chose $m=0.4$ for the mass of the scalar field, and $a=10$ for the radius of curvature. The values of the Casimir energy density $\rho_{C}$ on a sphere of proper (geodesic) radius $d=3.90035 \ldots$ inside the fundamental polyhedron for each of these manifolds are shown in Figs 2, 4, 6, and 8. In all these cases it can be seen that there is a spontaneous generation of low multipolar components. As expected, the effect becomes weaker for increasing volume universes.

\section{ACKNOWLEDGMENTS}

D.M. would like to thank the Brazilian agency FAPESP for financial support. H.V.F. thanks the Brazilian agency CNPq for partial support. R.O. thanks FAPESP and CNPq for partial support.
[1] B. Doubrovine, S. Novikov, and A. Fomenko, Géométrie Contemporaine-Méthodes et Applications ( $2^{e}$ Partie), Première édition (Mir, Moscow, 1982).

[2] H.V. Fagundes, Astrophys. J. 291, 450 (1985).

[3] J. Levin, Phys. Rep. 365, 251 (2002).

[4] M. Lachièze-Rey and J.-P. Luminet, Phys. Rep. 254, 135 (1995).

[5] E. Gaussman, R. Lehoucq, J.-P. Luminet, J.-P. Uzan, and J. Weeks, Class. Quantum Grav. 18, 5155 (2001).

[6] I.Y. Sokolov, JETP Lett. 57, 617 (1993).

[7] A. de Oliveira-Costa and G.F. Smoot, Astrophys. J. 448, 477 (1995).

[8] B.F. Roukema, Class. Quantum Grav. 17, 3951 (2000).

[9] R. Lehoucq, M. Lachièze-Rey, and J.-P. Luminet, Astron. Astrophys. 313, 330 (1996).

[10] R. Lehoucq, J.-P. Luminet, and J.-P. Uzan, Astron. Astrophys. 344, 735 (1999).

[11] G.I. Gomero, A.F.F. Teixeira, M.J. Rebouças, and A. Bernui, Int. J. Mod. Phys. D 11, 869 (2002).

[12] H.V. Fagundes and E. Gausmann, Phys. Lett. A 261, 235 (1999).

[13] N.J. Cornish, D. Spergel, and G. Starkman, Class. Quantum Grav. 15, 2657 (1998).

[14] G.I. Gomero, M.J. Rebouças, and R. Tavakol, Class. Quantum Grav. 18, L145 (2001).

[15] N.J. Cornish, D. Spergel, and G. Starkman, Phys. Rev. Lett. 77, 215 (1996).
[16] N.L. Balazs and A. Voros, Phys. Rep. 143, 109 (1986).

[17] J. Levin and J.D. Barrow, Class. Quantum Grav. 17, L61 (2000).

[18] D. Müller, H.V. Fagundes, and R. Opher, Phys. Rev. D 63, 123508 (2001).

[19] D. Müller and H.V. Fagundes, gr-qc/0205050.

[20] M. Bordag, U. Mohideen, and V.M. Mostepanenko, Phys. Rep. 353, 1 (2001).

[21] B.A. Dubrovin, A.T. Fomenko, and S.P. Novikov, Modern Geometry-Methods and Applications (Part 1), 2nd ed. (Springer-Verlag, New York, 1992).

[22] J. Schwinger, Phys. Rev. 82, 664 (1951).

[23] B.S. De Witt, Phys. Rep. 19, 296 (1975).

[24] S.M. Christensen, Phys. Rev. D 17, 946 (1978).

[25] S.M. Christensen, Phys. Rev. D 14, 2490 (1976).

[26] A.A. Grib, S.G. Mamayev, and V.M. Mostepanenko, Vacuum Quantum Effects in Strong Fields (Friedmann Laboratory Publishing, St. Petersburg, 1994).

[27] J. Weeks, SNAPPEA: A computer program for creating and studying hyperbolic 3-manifolds, freely available at site 〈http:// www.northnet. org/weeks $\rangle$.

[28] J.R. Weeks, Ph.D. thesis, Princeton University, 1985.

[29] S.V. Matveev and A.T. Fomenko, Russ. Math. Surveys 43, 3 (1988).

[30] W.P. Thurston, Bull. Am. Math. Soc. 6, 357 (1982).

[31] L.A. Best, Can. J. Math. 23, 451 (1971).

[32] C. Weber and H. Seifert, Math. Z. 37, 237 (1933). 